\title{
Update on Pharmacotherapy of Alcohol use disorder
}

\author{
Wole Akosile, MBBS, MBBS, MPH, Cert Addiction Psych, FRANZCP FAChAM
}

School of Medicine .Griffith university Queensland

\section{Background}

- Global burden of disease estimate that globally, there were between 63.5 [GBD 2010] and 95 million cases [GBD 2015] of AUD in 2015.

- 137,500 AUD related deaths [GBD 2015],

- 6.3 million years lived with disability [GBD 2015] and

- 112 million disability-adjusted life years [GBD 2015].

Definition of Alcohol use disorder by NIDA

- Alcohol use disorder is defined as a chronic, relapsing brain disease that is characterized by compulsive alcohol seeking and use, despite harmful consequences.

- It is considered a brain disease because alcohol changes the brain; it changes its structure and how it works. These brain changes can be long lasting and can lead to many harmful, often self-destructive, behaviours.

- Without a pharmacological adjunct to psychosocial therapy, the clinical outcome is poor, with up to $70 \%$ of patients resuming drinking within 1 year (Beckeret al).

\section{Alcohol Pharmacotherapy}

\section{- Naltrexone}

- Naltrexone is an opioid receptor antagonist that is thought to;

- Reduces the reward,

- Reduces the excitement associated with drinking alcohol and the related cues in the environment (anticipatory excitement)

- First line treatment

- Efficacy demonstrated in the combined study by Anton et al 2006

- Naltrexone is contraindicated in individuals with liver toxicity.

- Patients are often started on a half tablet $(25 \mathrm{mg})$ daily for the first 3-5 days to minimise adverse effects.

- There are no specific ill effects from alcohol consumption during treatment and patients do not need to be advised to stop therapy if they relapse.

- It has a slightly larger effect size than Acamprosate, but has more adverse effects including headache, nausea, lethargy and dysphoria. These effects are usually transient and rarely lead to cessation of therapy.

- Usually not prescribed in clients with chronic pain disorder and advanced liver disease.

- A Cochrane systematic review and meta-analysis, showed that treatment with naltrexone significantly reduced the risk of a return to heavy drinking to $83 \%$ of the placebo rate with a NNT of nine

\section{ACAMPROSATE}

- Acamprosate is a structural analogue of gamma aminobutyric acid (GABA).

- It is thought to work by affecting calcium channels and modifying transmission along GABA and glutamine pathways in the brain.

- This may result in decreased positive reinforcement of alcohol intake and withdrawal cravings.

- There is some evidence of neuro-protection.

- Five previous meta-analyses concluded that abstinence was significantly higher with Acamprosate.

- Considered first line in Australia

- The recommended dose is two $333 \mathrm{mg}$ tablets, three times a day for individuals over $60 \mathrm{~kg}$.

- Very well tolerated.
- The most common adverse event is transient diarrhoea and flatulence.

- Limited interaction with alcohol or drugs commonly prescribed in people living with AUD such as antidepressants, anxiolytics, disulfiram, naltrexone and neuroleptics.

- It can be given to patients with liver dysfunction.

- NNT) to achieve continuous abstinence was 7.8 at 6 months and 7.5 at 12 month $\{$ Mann et al $\}$

Disulfiram

- Disulfiram (Antabuse) interferes with the degradation of alcohol.

- Resulting in the accumulation of acetaldehyde.

- Alcohol is broken down in the liver by the enzyme alcohol dehydrogenase to acetaldehyde.

- The enzyme acetaldehyde dehydrogenase converts acetaldehyde to the harmless acetic acid

- Disulfiram blocks the enzyme acetaldehyde dehydrogenase

- After alcohol intake under the influence of disulfiram, the concentration of acetaldehyde in the blood may be 5 to 10 times higher than that found during metabolism of the same quantity of alcohol alone.

- Acetaldehyde is one of the major causes of the symptoms of "hangover",

- This produces a severe negative reaction to alcohol intake. Symptoms include flushing of the skin, accelerated heart rate, shortness of breath (Disulfiram reaction)

- The utility and effectiveness of disulfiram are considered limited because; Compliance is generally poor, not for emotionally unstable clients, not for clients who are "tunnel visioned" during crisis. Prescription must be done under expert guidance. Disulfiram may cause peripheral neuropathy

BACLOFEN

- Therefore, as a GABAB receptor agonist, baclofen is a biologically plausible therapeutic in the prevention of progression of AUDs.

- Baclofen can be used in patients with established liver disease because it is predominantly excreted via the kidneys.

- Efficacy in heavy drinkers established in the (ALPADIR study) and BACLOVILLE study.

- Different meta-analysis and RCTS have shown mixed reviews (Addolorato et al)

- However dose dependent effectiveness is consistent.

Topiramate

- A sulfamate-substituted monosaccharide related to fructose.

- An antiepileptic with neuroprotective properties.

- It reduces the rewarding effects of acute alcohol use by suppressing dopamine release, and normalises dopamine activity in chronic alcohol use.

- This reduces cravings for alcohol and withdrawal symptoms

- Topiramate is a mood stabiliser.

- It is useful in clients with co-morbidity e.g. bipolar disorder, borderline personality disorder and post-traumatic stress disorder (most individuals living with AUD have co-morbidity).

- Topiramate may be viewed as a way to address multiple disorders with one drug.

- Adverse effects are; Dizziness, Paraesthesia, Psychomotor slowing, Memory or concentration impairment, Weight loss. A syndrome consisting of acute myopia associated with secondary angle closure glaucoma has occasionally been reported. If there are sudden vision changes, eye pain or redness then topiramate should be ceased and medical review arranged.

Special mention

- Nalmefene (Opioid modulator/receptor blocker) It is approved in several European countries for treatment of AUD despite limited evidence from previous RCT's

(18mg daily).

- $\quad \mathrm{N}$-acetylcysteine Tomoko et al 2018

- Metadoxine (pyridoxal L-2-pyrrolidone-5-carboxylate) 